\title{
Epitaxy of Mn on Si(001): Adsorption, surface diffusion, and magnetic properties studied by density-functional theory
}

\author{
Mahbube Hortamani, ${ }^{1}$ Hua Wu, ${ }^{1,2}$ Peter Kratzer, ${ }^{1,3}$ and Matthias Scheffler ${ }^{1,4}$ \\ ${ }^{1}$ Fritz-Haber-Institut der Max-Planck-Gesellschaft, Faradayweg 4-6, D-14195 Berlin-Dahlem, Germany \\ ${ }^{2}$ II. Physikalisches Institut, Universität zu Köln, Zülpicher Strasse 77, 50937 Köln, Germany \\ ${ }^{3}$ Fachbereich Physik, Universität Duisburg-Essen, Lotharstrasse 1, D-47048 Duisburg, Germany \\ ${ }^{4}$ Department of Chemistry and Biochemistry, University of California Santa Barbara, Santa Barbara, California 93106-9510, USA
}

(Received 15 August 2006; published 7 November 2006)

\begin{abstract}
Ultrathin films of manganese silicides on silicon are of relevance as a possible material system for building spintronics devices with silicon technology. In order to achieve insight into epitaxial growth of such films on $\mathrm{Si}(001)$, total-energy calculations are presented using density-functional theory and the full-potential augmented plane wave plus local orbital method. For adsorption of a single $\mathrm{Mn}$ atom on $\mathrm{Si}(001)$, we find that binding at the subsurface sites below the Si surface dimers is $\sim 0.9 \mathrm{eV}$ stronger than on-surface adsorption. There is an energy barrier of only $0.3 \mathrm{eV}$ for adsorbed Mn to go subsurface, and an energy barrier of $1.2 \mathrm{eV}$ for the reverse process. From the calculated potential-energy surface for the Mn adatom, we conclude that the most stable site on the surface corresponds to the hollow site where Mn is placed between two Si surface dimers. For on-surface diffusion, both along and perpendicular to the $\mathrm{Si}$ dimer rows, the $\mathrm{Mn}$ atoms have to overcome energy barriers of $0.65 \mathrm{eV}$. For deposition of 0.5 monolayers (ML) or more, we find that the $\mathrm{Si}$ dimers of the $\mathrm{Si}(001)$ surface are broken up, and a mixed $\mathrm{MnSi}$ layer becomes the energetically most favorable structure. For coverages above $1 \mathrm{ML}$, the lowest-energy structure changes to a full Mn subsurface layer, capped by a layer of $\mathrm{Si}$ adatoms. We identify this transition with the onset of Mn-silicide formation in an epitaxially stabilized CsCl-like crystal structure. Such MnSi films are found to have sizable magnetic moments at the Mn atoms near the surface and interface, and ferromagnetic coupling of the Mn magnetic moments within the layers. Layer-resolved electronic densities of state are presented that show a high degree of spin polarization at the Fermi level, up to $45 \%$ and $27 \%$ for films with two or three Si-Mn layers, respectively.
\end{abstract}

DOI: 10.1103/PhysRevB.74.205305

PACS number(s): 68.43.Bc, 68.55.Ac, 75.70.-i

\section{INTRODUCTION}

Recently there has been growing interest in magnetic materials in semiconductor technology. The basic idea behind research in this field (typically called spintronics or magnetoelectronics) is the expectation that the spin of the electrons, in addition to their charge, should be used to process and store information. ${ }^{1,2}$ From a technological point of view, it would be highly desirable to grow heterostructures made from a ferromagnetic material and silicon. This goal can be achieved essentially in two ways, either by depositing a structurally well-defined thin film of a ferromagnetic metal on silicon, or by turning silicon into a magnetic semiconductor. We note that deposition of Mn on Si surfaces plays an important role for both approaches: Recently, it has been shown theoretically that ultrathin $\mathrm{MnSi}$ films on $\mathrm{Si}(001)$ display ferromagnetic (FM) behavior, as well as a high degree of spin polarization of carriers at the $\mathrm{MnSi} / \mathrm{Si}(001)$ interface. ${ }^{3,4}$ The possibility to grow strongly doped $\mathrm{Mn}: \mathrm{Si}$, which could possibly be ferromagnetic, in analogy to $\mathrm{Mn}: \mathrm{Ge},{ }^{5}$ has also been explored theoretically. ${ }^{6-8}$ Experimentally, it has recently been shown that a ferromagnetic phase with a Curie temperature above room temperature can be formed by $\mathrm{Mn}$ ion implantation into $\mathrm{Si}^{9}{ }^{9} \mathrm{An}$ interesting alternative are heterostructures of $\mathrm{Si}$ with Heusler alloy films, ${ }^{4}$ e.g., $\mathrm{Co}_{2} \mathrm{MnSi}$ or $\mathrm{Co}_{2} \mathrm{MnGe}$. These materials are not only ferromagnetic at room temperature, but also display a high spin polarization of carriers at the Fermi energy. Band structure theory predicts them to be magnetic half metals, i.e., ideally up to $100 \%$ spin polarization could be possible. Both these properties make heterostructures of Heusler alloys on silicon promising for efficient spin injection. For fabricating all the above-mentioned structures, a better theoretical understanding of the adsorption, diffusion, and nucleation of $\mathrm{Mn}$ on the silicon surface is desirable. In order to identify the elementary growth processes that determine the interface quality, we need information about the diffusion pathways of $\mathrm{Mn}$ on Si and their energy barriers.

Transition-metal silicides have been studied intensively, in particular $\mathrm{CoSi}_{2}$ and $\mathrm{TiSi}_{2} \cdot{ }^{10,11}$ However, for Mn-silicide films on Si only a few experimental studies have been performed thus far. Most experiments are concerned with $\mathrm{Mn} / \mathrm{Si}(111)$, where film growth with and without a Bi surfactant layer, ${ }^{12-15}$ surface structural phase transitions, ${ }^{16,17}$ as well as a non-metal-to-metal phase transition in 5-10 ML thick films ${ }^{18,19}$ have been reported. On Si(001), thin films of both metallic $\mathrm{MnSi}$ and semiconducting $\mathrm{MnSi}_{1.7}$ have been prepared by solid-phase reaction. ${ }^{20}$ In vapor-phase epitaxy of $\mathrm{Mn}$ on $\mathrm{Si}(001)$, first an unstructured film is created that transforms on annealing into three-dimensional islands..$^{21,22}$ The two types of islands observed, one with pancakelike shape and one with hutlike shape, were attributed to $\mathrm{MnSi}$ and $\mathrm{Mn}_{5} \mathrm{Si}_{3}$ nanocrystallites. ${ }^{21}$ Moreover, $\mathrm{Mn}_{x} \mathrm{Si}_{1-x}$ films with Mn concentration by far higher than the equilibrium solubility of $\mathrm{Mn}$ in Si have been created by molecular beam epitaxy on $\mathrm{Si}(001) .{ }^{23}$ From the theoretical side, Mn adsorption on $\mathrm{Si}(001)$ has been addressed in a recent study. Information about energy barriers and magnetic moments of Mn adatoms 
have been reported, ${ }^{6,7}$ with particular focus on Mn-doped Si as a possible ferromagnetic semiconductor. ${ }^{8}$ Here, we extend these investigations to systematic studies of the potential energy surface for Mn diffusion, to higher coverages of Mn, and to MnSi thin films on $\mathrm{Si}(001)$.

This paper is organized as follows: First, we investigate the behavior of single $\mathrm{Mn}$ adatoms on $\mathrm{Si}(001)$. Subsequently, we discuss ordered submonolayer films, their stability, and the magnetic and electronic properties of multilayer $\mathrm{MnSi}$ films. Finally, a summary and an assessment of the applicability of $\mathrm{Mn} / \mathrm{Si}$ as a system for spintronics applications is attempted.

\section{CALCUlations}

We employ density-functional theory (DFT) to determine the atomic structure, the relative stability, and the magnetic properties of numerous structures that occur during adsorption, bulk incorporation, and thin film growth of $\mathrm{Mn}$ on $\mathrm{Si}(001)$. The full-potential augmented plane-wave plus localorbital method ${ }^{24}$ is used for the calculations. We employed the generalized gradient approximation (GGA-PBE96) ${ }^{25}$ for the exchange-correlation potential, since it has been demonstrated $^{26}$ and confirmed by our calculations that it gives a much better description for bulk Mn than the localspin-density approximation (LSDA). Our test calculations show that the LSDA ground state is nonmagnetic (NM) and the lattice constant $(a=3.46 \AA)$ is $7 \%$ smaller than the experimental value $(3.72 \AA)$. In contrast, the GGA calculations for the fcc-Mn give the correct AFM ground state with $a$ $=3.59 \AA(3.5 \%$ smaller than the experimental value $)$ and a local spin moment of $1.9 \mu_{\mathrm{B}}$, well comparable with the experimental value of about $2.3 \mu_{\mathrm{B}}$.

A slab geometry is used to model the $\mathrm{Si}(001)$ surface consisting of eight (or, in some cases, ten) layers of Si atoms. $\mathrm{Mn}$ adatoms are placed on top and bottom surfaces of the slab to preserve the inversion symmetry of the supercell. We define $\theta_{\mathrm{Mn}}=1 \mathrm{ML}$ (monolayer) coverage of Mn by having a space-filling arrangement of two Mn adatoms per (1 $\times 1$ ) unit cell of the $\mathrm{Si}(001)$ surface (on either side of the slab). For Mn coverages $\leqslant 1 / 2 \mathrm{ML}$, the calculations were performed in a $p(2 \times 2)$ unit cell using a surface reconstruction with alternatingly buckled Si dimers. The periodic supercell contains $32 \mathrm{Si}$ atoms in an eight-layer slab of $\mathrm{Si}$, plus two to eight $\mathrm{Mn}$ atoms, according to the Mn coverage. The slabs are separated by a vacuum region of $16.4 \AA$. The Brillouin zone sampling in this case is done by a set of eight $\mathbf{k}$ points in the irreducible part of the Brillouin zone, derived from a $4 \times 4 \times 1 \mathbf{k}$ point mesh. With increasing the unit cell to $(4 \times 2)$, the number of $\mathbf{k}$ points are decreased to $2 \times 4$ $\times 1$ points. In all calculations, the muffin-tin sphere radius is set $1.11 \AA$ for both $\mathrm{Mn}$ and $\mathrm{Si}$, and cutoff energy for the plane-wave expansion in the interstitial region is $13.8 \mathrm{Ry}$. Such rather low cutoff energy is appropriate because of using additional local orbitals as basis functions. ${ }^{27}$ All $\mathrm{Mn}$ and $\mathrm{Si}$ atoms except for the two central-layer $\mathrm{Si}$ atoms were relaxed until all atomic forces are smaller than $0.03 \mathrm{eV} / \AA$. For the calculation of thin film structures with $\theta_{\mathrm{Mn}}>1 \mathrm{ML}$, a (1 $\times 1)$ unit cell is used, since the surface dimerization is lifted

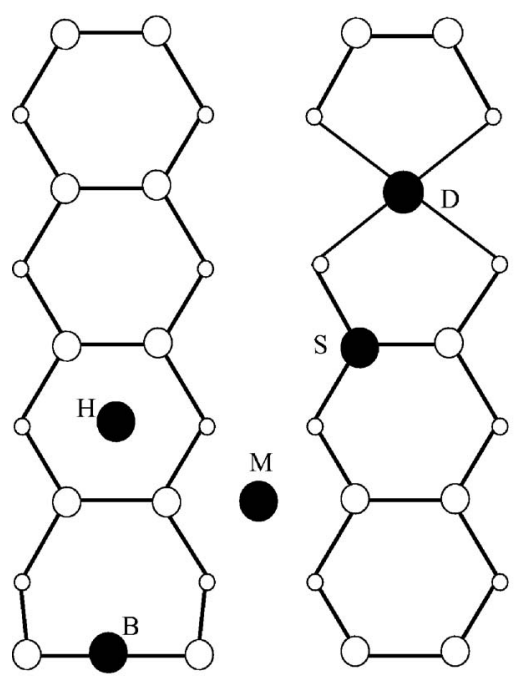

(a)

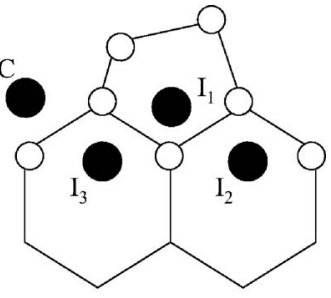

(b)

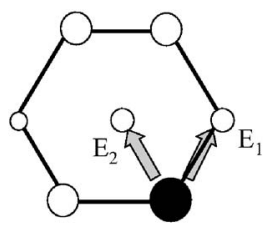

(c)
FIG. 1. (a) Schematic top view of the Si(001) surface. Filled circles indicate $\mathrm{Mn}$ and white circles $\mathrm{Si}$ atoms. (a) Top view of various binding sites for a $\mathrm{Mn}$ adatom on the surface in the dimer vacancy $(\mathrm{D})$, the hollow site $(\mathrm{H})$, the substitutional $(\mathrm{S})$, the dimer long bridge site $(\mathrm{M})$, and the dimer short bridge site (B). (b) Side view of adsorption site on sub-surface in the interstitial $\left(\mathrm{I}_{1}\right)$, the third-layer subsurface $\left(\mathrm{I}_{2}, \mathrm{I}_{3}\right)$ site, and the cave site $(\mathrm{C})$. (c) Top view illustrating substitutional adsorption of $\mathrm{Mn},\left(\mathrm{E}_{1}, \mathrm{E}_{2}\right)$; the arrows indicate two alternative positions for the expelled $\mathrm{Si}$ atom.

at $\theta_{\mathrm{Mn}}>1 / 2 \mathrm{ML}$ (see below). Here, a $10 \times 10 \times 1 \mathrm{k}$-point mesh for Brillouin zone integration and a vacuum region of $10-11 \AA$ are used.

The numerical accuracy of the present calculations has been checked for the clean $\mathrm{Si}(001)$ surface using a higher cutoff energy. It was found that the surface energy is converged within $0.01 \mathrm{eV}$ per $(1 \times 1)$ cell. Moreover, calculations for $\mathrm{MnSi}$ with different choice of the muffin-tin radii showed that changes are $<0.02 \mathrm{eV}$ per $(1 \times 1)$ cell. ${ }^{4}$ For the relative stability of structures with the same composition but different geometries and/or magnetic order, the computational error is even less, only a few millielectron volts, due to error cancellation in energy differences calculated with identical k-point set and energy cutoff.

All magnetic moments cited in this work are calculated by integrating the spin-up and spin-down charge densities over the muffin-tin sphere of the corresponding atom. The limited range of integration may result in a slight underestimation of magnetic moments. For instance, the magnetic moment of a free $\mathrm{Mn}$ atom within the muffin-tin sphere is evaluated to be $4.6 \mu_{\mathrm{B}}$, almost $8 \%$ smaller than the value of $5 \mu_{\mathrm{B}}$ expected from Hund's rule.

\section{RESULTS AND DISCUSSION}

\section{A. Mn adatom binding sites and diffusion}

Information about the binding sites of a Mn adatom on the $\mathrm{Si}(001)$ surface (cf. Fig. 1), as well as surface diffusion, is obtained from DFT calculations of the potential energy surface (PES) of a Mn atom, whose position is denoted by 


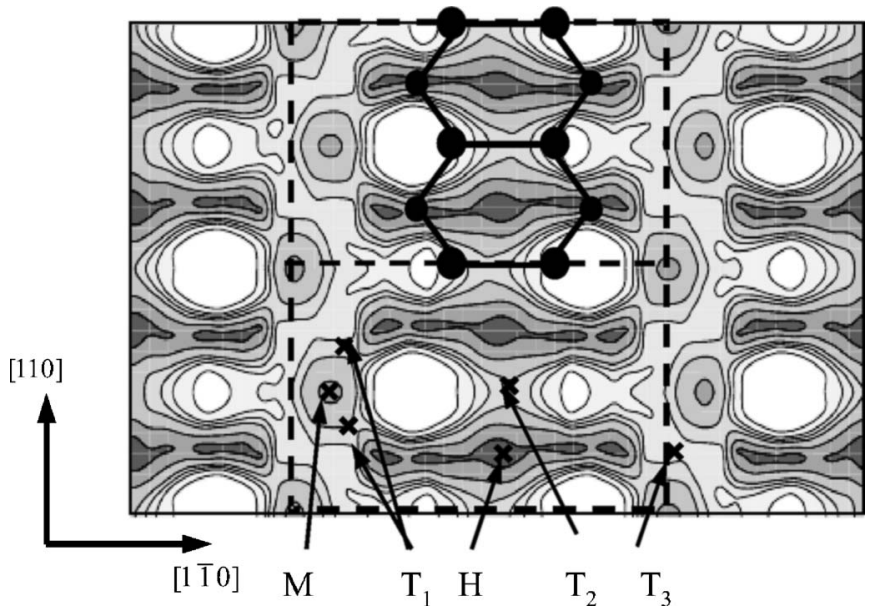

FIG. 2. The potential energy surface (PES) of a Mn atom on $\mathrm{Si}(001)$. The diffusion barriers for hopping between on-surface minima $\mathrm{H}$ are $0.65 \mathrm{eV}$. Diffusion along [1]10] (between Si dimer rows) occurs via transition state $\mathrm{T}_{3}$. Diffusion along [110] proceeds either through transition state $T_{2}$ or through transition state $T_{1}$ and intermediate $\mathrm{M}$.

$\mathbf{R}_{\mathrm{Mn}}=\left(X_{\mathrm{Mn}}, Y_{\mathrm{Mn}}, Z_{\mathrm{Mn}}\right)$. The PES is defined by relaxing both the height $Z_{\mathrm{Mn}}$ of the $\mathrm{Mn}$ adatom from far above the surface (for a set of fixed lateral coordinates $X_{\mathrm{Mn}}, Y_{\mathrm{Mn}}$ of the Mn atom), as well as all coordinates of the substrate atoms, starting from their positions on the clean surface

$$
E_{\mathrm{ad}}\left(X_{\mathrm{Mn}}, Y_{\mathrm{Mn}}\right)=\min _{\mathbf{R}} \min _{\mathrm{Z}_{\mathrm{Mn}}} E_{\text {system }}^{\mathrm{tot}}\left(\mathbf{R}, \mathbf{R}_{\mathrm{Mn}}\right)-E^{\text {slab }}-E^{\mathrm{Mn}} .
$$

Here $\mathbf{R}$ denotes the position of the substrate atoms, and $E^{\mathrm{Mn}}$ is the energy of a free $\mathrm{Mn}$ atom in its ground state according to Hund's rule, i.e., with a magnetic moment of $5 \mu_{\mathrm{B}}$. The total energy of the clean slab, $E^{\text {slab }}$, is obtained by a separate calculation for the clean $\mathrm{Si}(001)$ surface, for which we also obtained the bond lengths of the buckled Si dimers, $2.38 \AA$ and their buckling angles, $18^{\circ}$, in good agreement with literature values. ${ }^{28}$ Furthermore, we verified that this surface has two surface state bands lying in the bulk band gap, which are due to the dangling bonds at the Si surface dimers.

With this definition, $E_{\text {ad }}$ reflects the adsorption energy of a Mn adatom on $\mathrm{Si}(001)$. Local minima in the PES constitute (meta-)stable binding positions of the adatom, and saddle points correspond to transition states for adatom diffusion. Energy barriers for diffusion are calculated as differences between the transition state energy and the energy at the minimum of the PES which describes the initial state of the process. We note that some binding sites and transition states may not be contained in a so-defined PES, e.g., subsurface sites that cannot be reached directly (because they are behind an energy barrier) by relaxing the adatom from above the surface. In fact, we find that a subsurface interstitial site below the $\mathrm{Si}$ dimer, $\mathrm{I}_{1}$, yields the strongest binding for $\mathrm{Mn}$ atoms, with an adsorption energy of $3.8 \mathrm{eV}$. In Sec. III B, we investigate how an $\mathrm{Mn}$ atom can reach this subsurface site.

Figure 2 shows the potential-energy surface for a Mn adatom on the $\mathrm{Si}(001)$ surface. We find two local minima: The $\mathrm{Mn}$ atom binds most strongly at the hollow site (marked $\mathrm{H}$ in
TABLE I. Bond lengths, adsorption energy (in electron volts), and magnetic moment of $\mathrm{Mn}$ at low coverage, $\theta_{\mathrm{Mn}}=1 / 8$, for different adsorption sites shown in Fig. 1.

\begin{tabular}{cccc}
\hline \hline Site & $\begin{array}{c}\text { Si-Mn bond } \\
(\AA)\end{array}$ & $\begin{array}{c}E_{\text {ad }} \\
(\text { eV/atom })\end{array}$ & $\begin{array}{c}\text { Magnetic moment } \\
\left(\mu_{\mathrm{B}}\right)\end{array}$ \\
\hline $\mathrm{I}_{1}$ & $2.3-2.6$ & 3.80 & 2.4 \\
$\mathrm{D}$ & 2.5 & 4.29 & 2.9 \\
$\mathrm{I}_{3}$ & $2.4-2.5$ & 3.01 & 2.9 \\
$\mathrm{H}$ & 2.5 & 2.91 & 3.2 \\
$\mathrm{~S}$ & 2.4 & 2.86 & 3.6 \\
$\mathrm{~B}$ & $2.5-2.7$ & 2.72 & 3.9 \\
$\mathrm{E}_{1}$ & $2.3-2.4$ & 2.69 & 3.0 \\
$\mathrm{M}$ & 2.4 & 2.63 & 4.1 \\
$\mathrm{E}_{2}$ & $2.3-2.4$ & 2.36 & 2.9 \\
$\mathrm{C}$ & 2.4 & 2.69 & 3.3 \\
\hline \hline
\end{tabular}

Fig. 1), also called the pedestal site by other authors, ${ }^{6}$ located between two Si surface dimers. At this site, the Mn adatom makes bonds with all four neighboring surface $\mathrm{Si}$ atoms, whereas the $\mathrm{Si}-\mathrm{Si}$ dimer bonds are elongated. Compared to the free Mn atoms, adsorption in the hollow site goes along with a considerable reduction of its magnetic moment to a value of $3.2 \mu_{\mathrm{B}}$ within the muffin-tin sphere. In addition, we find a weaker binding site ( $\mathrm{M}$ in Fig. 2), where the Mn adatom interacts with the dangling bond of the upper Si atom in the surface Si dimer.

Table I gives the data for adsorption at some highsymmetry sites of the PES, as well as for some other adsorption geometries [the interstitial $\left(\mathrm{I}_{1}\right)$, the third-layer subsurface $\left(I_{3}\right)$, the dimer vacancy $(D)$, the cave site $(C)$, the hollow site $(H)$, the substitutional $(S)$, the exchange $\left(E_{1}, E_{2}\right)$, and the dimer short bridge site (B), cf. Fig. 1]. We find that the cave site $\mathrm{C}$ is $0.22 \mathrm{eV}$ higher in energy than the hollow site. We note that the interatomic distances between $\mathrm{Si}$ and nearest Mn neighbors, $d_{\mathrm{Mn}-\mathrm{Si}}$, are $2.38-2.40 \AA$, which is within the range of bond distances known from Mn-monosilicide bulk.

For on-surface diffusion between two hollow sites along the Si dimer rows, there exist two low-energy pathways, one where the adatom passes the (still intact) $\mathrm{Si}$ dimer through saddle point $\mathrm{T}_{2}$, and another pathway via the minimum $\mathrm{M}$, passing twice through symmetry-equivalent saddle points $T_{1}$. For the first diffusion pathway, the energy barrier is about $0.65 \mathrm{eV}$ (see Fig. 2, lower left panel), while it is slightly lower $(0.55 \mathrm{eV})$ for the second pathway (see Fig. 2, lower right panel). For diffusion from one dimer row to the next, the $\mathrm{Mn}$ adatom needs to overcome a barrier of $0.65 \mathrm{eV}$ at $\mathrm{T}_{3}$ (see Fig. 2, lower left panel). In summary, the Mn diffusion is almost isotropic as long as only on-surface hopping processes are considered.

In the context of the epitaxial growth of Mn-doped $\mathrm{Si}$, incorporation of $\mathrm{Mn}$ at substitutional positions at the surface is particularly important. On the one hand, this process is likely to trigger silicide formation (see Sec. III D). On the other hand, it has long been known that substitutional Mn atoms in bulk $\mathrm{Si}$ act as acceptors ${ }^{29}$ with a large local magnetic moment, and hence, one could speculate that Si could 
be turned into a magnetic semiconductor if substitutional Mn incorporation with a high concentration could be achieved. We therefore studied the energetics of $\mathrm{Mn}$ replacing a $\mathrm{Si}$ atom of the Si surface dimer. If we take the chemical potential of bulk $\mathrm{Si}$ as energy reference, substitutional adsorption is energetically less favorable than adsorption into the subsurface interstitial site, $\mathrm{I}_{1}$, by $0.94 \mathrm{eV}$. In an attempt to estimate the energy barrier for a surface exchange process of Mn and $\mathrm{Si}$, we have calculated geometries where $\mathrm{Mn}$ occupies the Si site, while the replaced Si atom sits at different neighboring positions. Specifically, we investigate the possibilities that the exchanged $\mathrm{Si}$ atom could move to an asymmetric position between two Si dimers (marked as $\mathrm{E}_{1}$ in Fig. 1), or to the hollow site (marked as $E_{2}$ in Fig. 1). The adsorption energies for these two possibilities are 0.22 and $0.55 \mathrm{eV}$ lower than for $\mathrm{Mn}$ at the hollow site. Since the energies of these intermediate configurations can be considered as a lower bound for the energy barrier of substitution, we conclude that a $\mathrm{Mn}$ atom in the hollow site needs to overcome a barrier of at least $0.22 \mathrm{eV}$ to create a substitutional $\mathrm{Mn}$ site at the surface. However, once a Mn atom has reached the interstitial site $I_{1}$, the activation energy for creating a substitutional $\mathrm{Mn}$ is increased by $0.89 \mathrm{eV}$. The barrier for reaching the interstitial site from the hollow site is only $0.3 \mathrm{eV}$ (see Sec. III B). Hence, we conclude that substitutional and interstitial adsorption of $\mathrm{Mn}$ are competing processes.

Finally, we investigate the role of surface imperfections on Mn adsorption. An abundant defect on a $\mathrm{Si}(001)$ surface is the missing-dimer defect. ${ }^{30} \mathrm{In}$ order to describe such defects, we use a larger unit cell having either $(2 \times 4)$ or $(4$ $\times 2$ ) symmetry. In the first case, we model a $\mathrm{Si}$ dimer row, consisting of blocks of three $\mathrm{Si}$ dimers, interrupted by a dimer vacancy. In the second case of the $(4 \times 2)$ unit cell, the surface consists of continuous rows of Si dimers, alternating with rows where a Si dimer and a dimer vacancy follow each other. Interestingly, we find that the second type of dimer vacancies (single dimers alternating with vacant sites in a row) is more stable by $0.48 \mathrm{eV} /$ unit cell. The adsorption energy of $\mathrm{Mn}$ at the dimer vacancy is calculated to be $4.29 \mathrm{eV}$ for this $(4 \times 2)$ unit cell. Thus, our calculations show that an Mn adatom binds very strongly to this site, with a binding energy $1.4 \mathrm{eV}$ larger than the binding energy at the hollow site. The Mn atom occupying the dimer vacancy comes very close to the surface and sits at about the same height as the Si dimers. It is sixfold coordinated to Si and possesses a magnetic moment of $2.9 \mu_{\mathrm{B}}$. Consequently, the formation of a Mn-decorated missing-dimer defect becomes exothermic, if we assume that the two expelled $\mathrm{Si}$ atoms move to kink sites at steps (i.e., to a reservoir at the chemical potential of bulk $\mathrm{Si}$ ).

Dalpian et al. ${ }^{6}$ have performed a similar study, using ultrasoft pseudopotentials and $(4 \times 4)$ unit cell to describe the surface. Our results are in good agreement with theirs, except for the energy barrier between the hollow site and the subsurface interstitial site, which they estimated to be $0.69 \mathrm{eV}$, however without a systematic determination of the transition state geometry. Here, we find that a barrier height of $0.3 \mathrm{eV}$ from our calculating of the PES (see Sec. III B). Likewise, the magnetic moments obtained in the pseudopotential calculations ${ }^{7}$ are in fair agreement with ours, except for the second layer interstitial site, where the pseudopotential calculation yields a considerably smaller magnetic moment $\left(0.92 \mu_{\mathrm{B}}\right)$ than our all-electron calculations $\left(2.4 \mu_{\mathrm{B}}\right)$. One possible reason for the discrepancy could be that the pseudopotential calculations were performed by constraining the total spin of per unit cell to $S=3 / 2$, thus precluding a high-spin state of the Mn atom. Moreover, we note that the magnitude of the magnetic moment may depend sensitively on core polarization effects, which we find to differ for $\mathrm{Mn}$ in different adsorption sites.

Comparing to recent calculations by Zhu et al. for $\mathrm{Mn} / \mathrm{Ge}(001),{ }^{5}$ we conclude that $\mathrm{Mn}$ shows very similar behavior on $\mathrm{Ge}(001)$ and on $\mathrm{Si}(001)$. In both cases, the calculations show that the adatom has a tendency to penetrate to the second-layer interstitial site via the hollow site, the latter being the most stable site on the surface. Zhu et al. ${ }^{5}$ report an energy barrier of $0.59 \mathrm{eV}$ for this process, and $1.22 \mathrm{eV}$ for the reverse process, which is comparable to our results for $\mathrm{Mn} / \mathrm{Si}(001)$. For $\mathrm{Mn}$ on $\mathrm{Ge}(001)$, the interstitial sites in deeper layers, as well as the surface substitutional site, are also found to be less stable than the second layer interstitial site, in agreement with the behavior that we find for $\mathrm{Mn} / \mathrm{Si}(001)$.

Similarly, calculations for $\mathrm{Mn} / \mathrm{GaAs}(001)$ identified the interstitial site below an As surface dimer to be the most stable site for $\mathrm{Mn}$ on $\operatorname{GaAs}(001) \cdot{ }^{31}$ It was found that the energy barrier for $\mathrm{Mn}$ to break the As dimer and penetrate to the subsurface site is only $0.2 \mathrm{eV}$ for this system. In contrast to $\mathrm{Si}$, for $\mathrm{GaAs}$ the cave site $\mathrm{C}$ was reported to be stable, and the energy barrier to reach this position was found to be negligible.

It is also interesting to compare with results for adsorption of other transition metals on $\mathrm{Si}(001)$. DFT calculations for the adsorption of $\mathrm{Ti}^{32} \mathrm{Co}^{10}$ and $\mathrm{Ni}^{33}$ on $\mathrm{Si}(001)$ have been published. For all transition metals studied, adsorption at the Si dimer vacancy shows the highest adsorption energy, in agreement with our results. For adsorption on the defect-free surface, some differences are noteworthy. Although $\mathrm{Co}^{10}$ and $\mathrm{Ni}^{33}$ show qualitatively similar behavior as we find for $\mathrm{Mn}$, substitutional adsorption of $\mathrm{Ti}$ (as part of the $\mathrm{Si}$ surface dimer) is energetically more favorable than any other adsorption site. ${ }^{32}$ It appears that the early transition metal Ti prefers low coordination and diffuses by an exchange mechanism.

\section{B. Mn subsurface adsorption and diffusion}

It is known from experiments that Mn in bulk silicon is a mobile impurity, which occupies preferentially interstitial sites. ${ }^{34,35}$ Therefore one might expect that subsurface sites, in addition to on-surface sites, play a role for Mn adsorption and surface diffusion, and we consider this possibility in this section.

The calculations show that the second-layer interstitial site below the $\mathrm{Si}$ dimer, marked $\mathrm{I}_{1}$ in Fig. 1 , with $3.8 \mathrm{eV}$ adsorption energy, is actually the most stable site, lower in energy than any surface site. A Mn atom in this site is located $2 \AA$ below the Si dimer, whose length increases to $2.8 \AA$, $\sim 17 \%$ more than the usual Si dimer bond length of $2.4 \AA$. At 

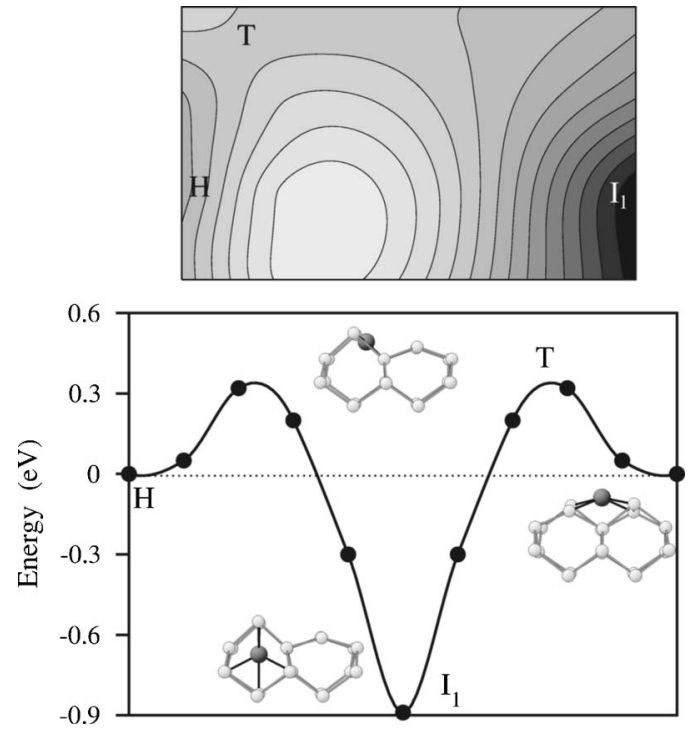

FIG. 3. PES and energy barrier for the penetration of Mn to the subsurface site $I_{1}$. The lower panel shows the energy barrier of $0.3 \mathrm{eV}$, and the geometries of the initial state $\mathrm{H}$, the transition state $\mathrm{T}$ and the final state $\mathrm{I}_{1}$ in side view, looking along the $[\overline{1} 10]$ direction.

the same time, this dimer becomes parallel to the surface and is lifted up by $1 \AA$. The neighboring Si dimer moves closer to the surface, and its bond length is slightly reduced, to $2.3 \AA$. In this atomic configuration, Mn forms nine bonds with the neighboring $\mathrm{Si}$ atoms with distances between 2.3 and $2.6 \AA$. Its magnetic moment is reduced compared to onsurface adsorption, to a value of $2.4 \mu_{\mathrm{B}}$.

Furthermore, we performed calculations for the thirdlayer interstitial sites marked by $\mathrm{I}_{2}$ and $\mathrm{I}_{3}$ in Fig. 1(b). These two sites have a different atomic environment due to the buckling of the Si surface dimers. Our calculations show that only the site below the lower $\mathrm{Si}$ dimer atom, $\mathrm{I}_{3}$, is a local minimum, while $\mathrm{Mn}$ placed in $\mathrm{I}_{2}$ spontaneously relaxes to site $I_{1}$. Our results thus indicate that the third-layer interstitial site is energetically less favorable than the second-layer interstitial site. Other calculations have arrived at the same result for $\mathrm{Mn} / \mathrm{Si}(001)^{6}$ and $\mathrm{Mn} / \mathrm{Ge}(001),{ }^{5}$ and have shown that the trend to lower adsorption energies with increasing depth below the surface extends to deeper subsurface layers and the bulk interstitial site.

Next we consider the question how the Mn adatom diffuses into the second layer site, i.e., how it reaches the interstitial site from the hollow site, which is the energetically lowest site on the surface. Determining the diffusion pathway requires special care because considerable relaxation of the neighboring Si atoms occurs along the pathway. For this

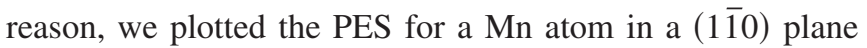
perpendicular to the surface, which intersects both the hollow and the interstitial sites. We fix the Mn atom at a set of positions in this plane, and relax the substrate $\mathrm{Si}$ atoms in each case. The resulting PES, spanned by [110] and [001] vectors, is displayed in Fig. 3. It is seen that for the most favorable pathway the $\mathrm{Mn}$ adatom first moves slightly upward away from the hollow site. Thereby, the surface Si

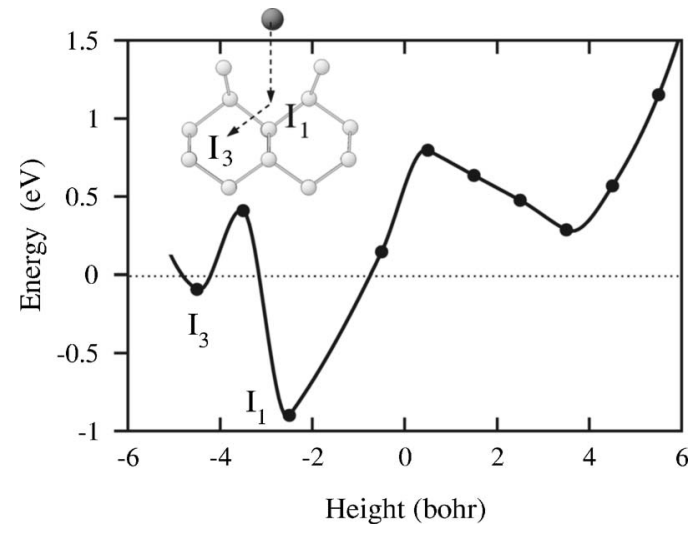

FIG. 4. PES and atomic structure for a Mn atom approaching the $\mathrm{Si}(001)$ surface from the vacuum. The $\mathrm{Mn}$ atom breaks the $\mathrm{Si}$ dimer and inserts itself via the stable subsurface site $I_{1}$ into the third-layer interstitial site $\mathrm{I}_{3}$. The energy barrier for the last step, from $\mathrm{I}_{1}$ to $\mathrm{I}_{3}$, is $\sim 1.3 \mathrm{eV}$.

dimer is elongated, thus giving room for the Mn atom to find its way to the subsurface interstitial site. The energy barrier for the penetration pathway $\mathrm{H}-\mathrm{T}-\mathrm{I}_{1}$, crossing the transition state $\mathrm{T}$, is $\sim 0.3 \mathrm{eV}$ measured from the hollow site, and $\sim 1.2 \mathrm{eV}$ for the reverse process (see Fig. 3, lower panel).

In addition, we consider the possibility that a $\mathrm{Mn}$ atom coming from the vacuum could directly reach the secondlayer interstitial site, by normal incidence and breaking of the $\mathrm{Si}$ dimer. For these calculations, we use a $c(4 \times 2)$ unit cell, to avoid spurious interactions between neighboring $\mathrm{Si}$ dimers being broken, which could occur if a $p(2 \times 2)$ cell would be used for simulating this process. The calculations show that the energy of this system first decreases when Mn approaches the surface, but then rises again by $\sim 0.5 \mathrm{eV}$ and goes through a maximum before decreasing finally to the binding energy in the interstitial site (Fig. 4). The intermediate increase in energy is associated with the Mn atom breaking the Si-Si dimer bond. However, the energy gained by the Mn atom during approaching the surface is more than sufficient to overcome this energy barrier. Hence, we conclude that a deposited $\mathrm{Mn}$ atom can reach the interstitial site both indirectly through the hollow site, or directly by breaking the Si dimer on impact, if the site and angle of impact are appropriate. We note that the possibility of such a direct process was also demonstrated by calculations for $\mathrm{Mn}$ on $\operatorname{GaAs}(001){ }^{31}$

For other transition metals on $\mathrm{Si}(001)$, such as $\mathrm{Ti}, \mathrm{Co}$, and $\mathrm{Ni}$, DFT calculations ${ }^{10,32,33}$ have also found the relatively stable subsurface adsorption site $I_{1}$. However, for Ti, substitutional adsorption is preferable over adsorption in the subsurface interstitial site. ${ }^{32}$ For $\mathrm{Ni}$, adsorption as a third-layer interstitial in site $I_{2}$, which we find to be unstable for $\mathrm{Mn}$, is even more favorable than the site $I_{1}$. For Co, however, the preference for the $I_{1}$ site is even more pronounced $(1.58 \mathrm{eV}$ lower than the $H$ site $)^{10}$ than for $\mathrm{Mn}(0.89 \mathrm{eV}$ lower $)$. Although $\mathrm{Mn}$ and $\mathrm{Co}$, forming strong bonds to $\mathrm{Si}$, can benefit in the $I_{1}$ site from the weakening of the Si surface dimer, bond formation to $\mathrm{Si}$ is considerably weaker both for the early transition metal $\mathrm{Ti}$ and the late transition metal Ni.

Our calculations show that there are high energy barriers for diffusion of $\mathrm{Mn}$ into deeper layers. To reach the third- 
layer site, Mn must overcome an energy barrier of $1.3 \mathrm{eV}$, measured from the second-layer interstitial site (Fig. 4). Therefore, it is concluded that Mn atoms, even after penetrating to the subsurface site, will diffuse mainly through the on-surface $(\mathrm{H})$ site, rather than through a bulk diffusion mechanism.

\section{Ordered submonolayer structures}

Next we study adsorption of Mn at higher coverage, in the range between $1 / 4$ and $1 \mathrm{ML}$. It is our goal to investigate how the adsorption energy and the magnetic moment of the $\mathrm{Mn}$ atom change with coverage. Although experiments ${ }^{21}$ find an inhomogeneous distribution of $\mathrm{Mn}$ on the surface, leading to island formation, we have to assume homogeneous, ordered structures for our supercell calculations. However, since the properties of interest depend mostly on the local coordination of the Mn atoms, the trends observed in our calculations are expected to carry over to inhomogeneous layers, too. With our choice of the $p(2 \times 2)$ cell, Mn coverages between 1/4 and $1 \mathrm{ML}$ corresponds to adsorption of two to eight $\mathrm{Mn}$ atoms per supercell.

For two Mn atoms, one possibility is the adsorption at adjacent interstitial and hollow sites. This geometry is found to be the most stable arrangement for a pair of Mn atoms in our calculations. Other configurations that can be realized by two $\mathrm{Mn}$ atoms in the $p(2 \times 2)$ cell corresponding to an infinite chain of Mn adatoms. Chains in the [110] direction (along the dimers rows) can be formed in two ways, either by occupation of all on-surface hollow sites, or by occupation of all subsurface interstitial sites. We find that the adsorption energy per $\mathrm{Mn}$ atom in an infinite chain is larger than for a single atom per unit cell for the hollow site, while for the interstitial site the infinite chain has a lower absorption energy compared to single $\mathrm{Mn}$ atoms. In other words, Mn adatoms in hollow sites interact attractively, whereas $\mathrm{Mn}$ atoms in the interstitial site show a repulsive interaction. The latter observation can be understood from the fact that $\mathrm{Mn}$ atoms in interstitial sites introduce tensile strain by widening the $\mathrm{Si}$ crystal lattice in their neighborhood, and hence, two interstitial $\mathrm{Mn}$ atoms repel each other through these strain fields. However, a chain of interstitial Mn atoms is still lower in energy than the chain of hollow site Mn atoms; i.e., the trend for occupying subsurface positions persists. Furthermore, we considered chains of on-surface $\mathrm{Mn}$ atoms in [1 $\overline{1} 0]$ direction perpendicular to the $\mathrm{Si}$ dimer rows, where alternately one $\mathrm{Mn}$ atom is in a hollow site, and one $\mathrm{Mn}$ atom is in a cave site. Although the adsorption energy per atom is less than the average adsorption energy of these two sites calculated separately (i.e., there is repulsive interaction), this chain is energetically less favorable than any chain of $\mathrm{Mn}$ atoms running in the [110] direction.

For a coverage of $3 / 8 \mathrm{ML}$, i.e., three Mn atoms per unit cell, we studied two possibilities: For on-surface adsorption, we consider a chain of $\mathrm{Mn}$ atoms in hollow sites, plus one $\mathrm{Mn}$ atom in a cave site. For subsurface adsorption, we consider a chain of interstitial $\mathrm{Mn}$ atoms, plus one $\mathrm{Mn}$ atom occupying a hollow site on the surface. As for the 1/4 ML case, the latter possibility, combining interstitial and hollow

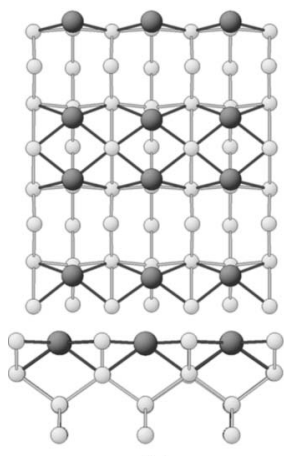

(a)

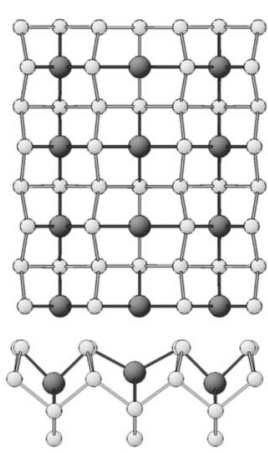

(b)

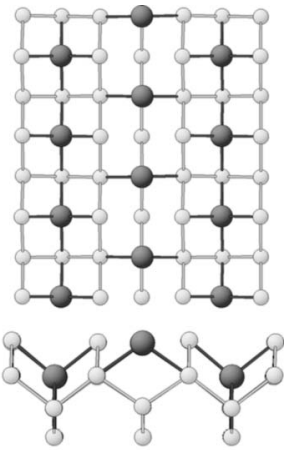

(c)
FIG. 5. Different possibilities for adsorption of 1/2 ML of Mn on $\mathrm{Si}(001)$, shown in top view (upper panels) and side view (lower panels). (a) Relaxed structures resulting from $\mathrm{Mn}$ in on-surface sites only, (b) in subsurface sites and cave site, and (c) in cave and hollow sites.

sites, is energetically more favorable. In both geometries, the Si dimers are still intact, albeit elongated.

For a coverage of $1 / 2 \mathrm{ML}$, we consider four Mn adatoms occupying both hollow sites and both cave sites of the unit cell. Starting from this geometry, the surface spontaneously relaxes to a configuration where each of the Mn atoms has four in-plane bonds to neighboring Si atoms, formerly being part of Si dimers, which have been broken up due to bonding to the $\mathrm{Mn}$ atoms. In addition, each $\mathrm{Mn}$ atom establishes two bonds to second-layer $\mathrm{Si}$ atoms. This structure has a $(1 \times 1)$ periodicity, the same as the underlying substrate. Its energy can be lowered slightly if two neighboring $\mathrm{Mn}$ atoms move toward each other along the [110] direction [see Fig. 5(a)]. Because of this relaxation, the symmetry of the surface reconstruction is lowered from $(1 \times 1)$ to $(1 \times 2)$, with the long side of the unit cell now along the direction of the former $\mathrm{Si}$ dimer row. Alternatively, we study the case of subsurface adsorption. This atomic configuration is obtained by relaxing a structure where $\mathrm{Mn}$ atoms are sitting in interstitial sites, and also at cave sites in the trenches between Si dimer rows, the latter $\mathrm{Mn}$ atoms either bonding to two third-layer $\mathrm{Si}$ atoms [see Fig. 5(b)], or sitting directly above one third-layer $\mathrm{Si}$ atom [see Fig. 5(c)]. From our calculations, we find that at 1/2 ML coverage, on-surface [Fig. 5(a)] and subsurface [see Fig. 5(b)] adsorption yields the same energies within numerical accuracy, whereas the mixed occupation of subsurface and on-surface sites shown in Fig. 5(c) gives an energy lower by $\sim 0.24 \mathrm{eV}$ per adatom.

On further increasing the number of $\mathrm{Mn}$ atoms in the mixed Mn-Si layer, the system has the tendency to adsorb the additional $\mathrm{Mn}$ atoms in top positions, leading to a mixed occupation of both subsurface and on-surface sites [see Fig. 6(c)] This is energetically more favorable than accommodating all $\mathrm{Mn}$ atoms in the subsurface layer [see Fig. 6(b)] or topmost layer [see Fig. 6(a)]. The configuration shown in Fig. 6(c) is also found to be slightly more favorable (by $\sim 0.06 \mathrm{eV}$ per $\mathrm{Mn}$ atom) than an alternative where Mn substitutes for the atoms in the subsurface layer. For mixed structures with $\theta=3 / 4 \mathrm{ML}$, we also calculate the energy difference between the two possibilities where the dense Mn layer is the subsurface layer rather than the topmost layer; 


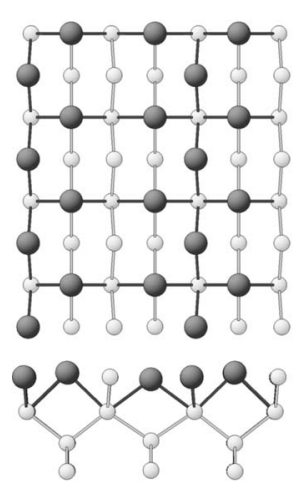

(a)

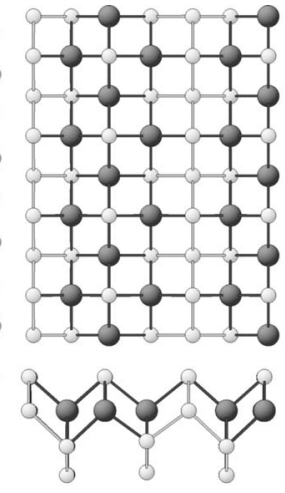

(b)

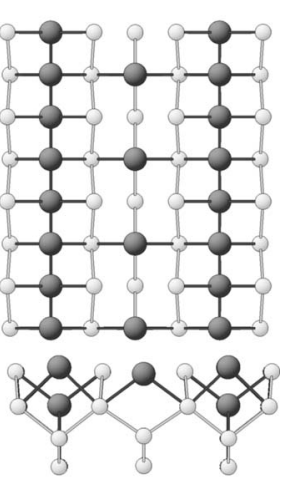

(c)

FIG. 6. Structures for adsorption of 3/4 ML of Mn on $\mathrm{Si}(001)$, shown in top view (upper panels) and side view (lower panels). (a) Structure resulting from adding $\mathrm{Mn}$ atoms in the topmost layer and relaxing (b) subsurface layer of $\mathrm{Mn}$ with $\mathrm{Si}$ capping layer and (c) structure derived from 1/2 ML Mn in on-surface sites plus 1/4 ML Mn in subsurface sites.

the former configuration is found to be higher in energy by $0.11 \mathrm{eV} / \mathrm{Mn}$.

For the geometries considered for coverages up to $1 / 2$ $\mathrm{ML}$, all $\mathrm{Mn}$ atoms are coordinated to Si atoms only, and their magnetic moments are found to be similar to those of single Mn atoms reported in Table I, about $3.2 \mu_{\mathrm{B}}$ for on-surface atoms, and about $2.4 \mu_{\mathrm{B}}$ for subsurface atoms. For the stable structure at $\theta=3 / 4 \mathrm{ML}$, Fig. 6(c), we encounter for the first time the situation that $\mathrm{Mn}$ atoms have $\mathrm{Mn}$ nearest neighbors. This leads to enhanced overlap of the Mn $d$ orbitals and raises the question about the dominant magnetic coupling mechanism between the Mn magnetic moments. From our calculations, we find antiferromagnetic ordering for the Mn magnetic moments in the same layer to be energetically preferable by $\sim 0.05 \mathrm{eV}$ per atom compared to ferromagnetic ordering. In the antiferromagnetically ordered structure, the magnetic moments at the $\mathrm{Mn}$ atoms in the topmost layer are $-3.5 \mu_{B}$ and $3.2 \mu_{B}$, respectively, while the magnetic moment of the $\mathrm{Mn}$ atom in the second layer is reduced to $-0.5 \mu_{B}$. For the slightly less stable ferromagnetic ordering, the magnetic moments are $3.5 \mu_{\mathrm{B}}, 3.3 \mu_{\mathrm{B}}$, and $1.3 \mu_{\mathrm{B}}$ (for the subsurface Mn atom), respectively.

Further increasing the coverage of $\mathrm{Mn}$ to $1 \mathrm{ML}$ amounts to filling both the subsurface and on-surface sites, resulting in two dense layers with mixed site occupation by $\mathrm{Mn}$ and $\mathrm{Si}$ atoms [cf. Fig 7(c)]. We find that adsorption in separate layers, i.e., one pure Mn monolayer capped by a dense Si layer [cf. Fig 7(b)], is energetically more favorable than two intermixed layers, or a pure overlayer structure [Fig. 7(a)]. However, formation of the former structure requires that the Mn atoms substitute for the second-layer $\mathrm{Si}$ atoms, and hence a site exchange between $\mathrm{Mn}$ and $\mathrm{Si}$ must take place, associated with an energy barrier. We consider the capped Mn layer as the starting point of silicide formation, while the structures discussed so far are considered as adsorbate layers of $\mathrm{Mn}$ on $\mathrm{Si}(001)$. Silicide films will be treated in more detail in the next section.

The general trend in adsorption of $1 / 8 \mathrm{ML}$ up to $1 \mathrm{ML}$ of $\mathrm{Mn}$ is summarized in Table II, quoting the average adsorp-

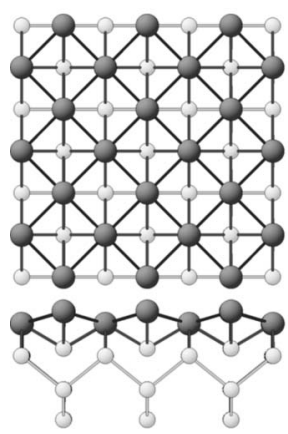

(a)

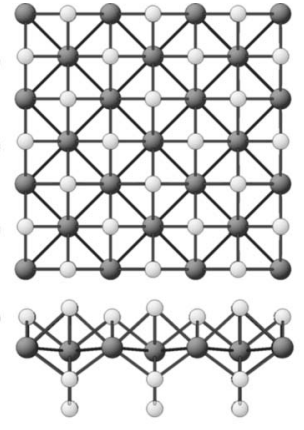

(b)

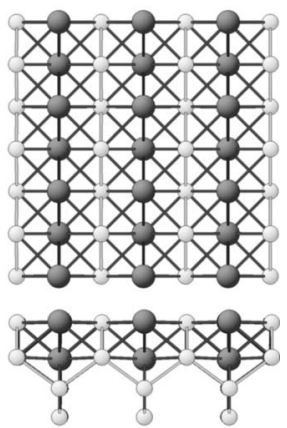

(c)
FIG. 7. Structures for adsorption of $1 \mathrm{ML}$ of $\mathrm{Mn}$ on $\mathrm{Si}(001)$, shown in top view (upper panels) and side view (lower panels). (a) Pure $\mathrm{Mn}$ in top-most layer, (b) a pure $\mathrm{Mn}$ film capped with a $\mathrm{Si}$ layer, and (c) mixed Mn-Si layers.

tion energy per Mn atom. The mixed occupation of subsurface and on-surface sites is found to be most favorable. Although the average adsorption energy drops from 3.8 to $3.44 \mathrm{eV}$ when raising the coverage to $1 / 4 \mathrm{ML}$, it remains roughly constant at $\sim 3.5 \mathrm{eV}$ for higher coverages up to 1 ML. Alternating occupation of subsurface and onsurface sites is identified as the preferred mechanism for building up $\mathrm{MnSi}$ clusters on $\mathrm{Si}(001)$. At $1 \mathrm{ML}$ of $\mathrm{Mn}$ or above, the system lowers its energy further by site exchange between $\mathrm{Mn}$ and Si leading to a complete Mn layer covered by $\mathrm{Si}$.

\section{Multilayer MnSi films}

For thin overlayers containing more than $1 \mathrm{ML}$ of $\mathrm{Mn}$, it is convenient to discuss their stability in terms of their formation energy. Since the $\mathrm{Si}$ dimer reconstruction is lifted already after deposition of $1 / 2 \mathrm{ML}$ of $\mathrm{Mn}$, it is appropriate to use a $\mathrm{Si}(001)(1 \times 1)$ unit cell to study thicker films. The formation energy is defined as

$$
E_{\text {form }}=\frac{1}{2}\left[E_{\text {total }}-N_{\mathrm{Si}} \mu_{\mathrm{Si}}-N_{\mathrm{Mn}} \mu_{\mathrm{Mn}}\right]-E_{\mathrm{Si}(001)}^{\mathrm{surf}},
$$

where $E_{\text {total }}, N$, and $\mu$ refer to total energy and the number of atoms per $(1 \times 1)$ supercell, and chemical potential of the

TABLE II. Average adsorption energies per Mn atom at various Mn coverages $\theta_{\mathrm{Mn}}$ with respect to bulk $\mathrm{Mn}$ and bulk Si. The left column is for all $\mathrm{Mn}$ atoms occupying on-surface $(\mathrm{H})$ sites, the middle column is for all $\mathrm{Mn}$ atoms in subsurface $\left(\mathrm{I}_{1}\right)$ sites, and the right column is for structures where subsurface $\left(\mathrm{I}_{1}\right)$ and on-surface (H) sites are alternatingly populated.

\begin{tabular}{cccc}
\hline \hline $\begin{array}{c}\text { Mn coverage } \\
(\mathrm{ML})\end{array}$ & $\begin{array}{c}\text { On surface } \\
(\mathrm{eV} / \mathrm{Mn})\end{array}$ & $\begin{array}{c}\text { Subsurface } \\
(\mathrm{eV} / \mathrm{Mn})\end{array}$ & $\begin{array}{c}\text { Sub+on surface } \\
(\mathrm{eV} / \mathrm{Mn})\end{array}$ \\
\hline$\theta_{\mathrm{Mn}}=1 / 8$ & 2.91 & 3.80 & - \\
$\theta_{\mathrm{Mn}}=2 / 8$ & 3.01 & 3.40 & 3.44 \\
$\theta_{\mathrm{Mn}}=3 / 8$ & 3.32 & 3.31 & 3.40 \\
$\theta_{\mathrm{Mn}}=4 / 8$ & 3.29 & 3.31 & 3.55 \\
$\theta_{\mathrm{Mn}}=6 / 8$ & 3.06 & 3.45 & 3.52 \\
$\theta_{\mathrm{Mn}}=1.0$ & 3.07 & 3.63 & 3.46 \\
\hline \hline
\end{tabular}




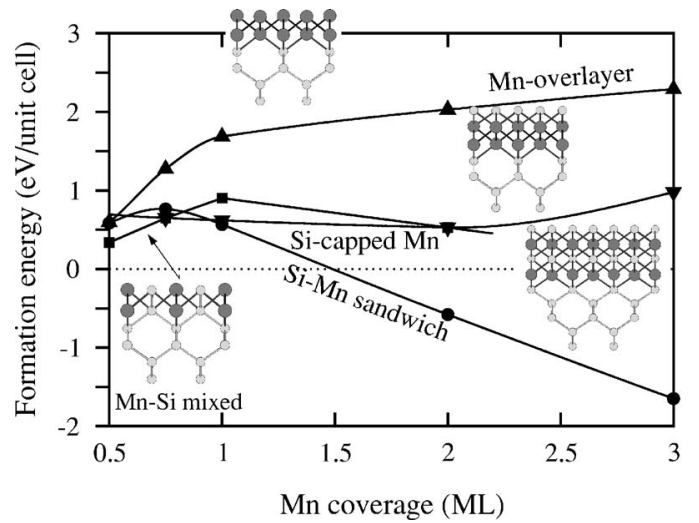

FIG. 8. Formation energy of Mn films and MnSi films in the $\mathrm{CsCl}$ crystal structure as a function of the Mn coverage. The zero of the energy scale refers to the surface energy of the clean reconstructed $\mathrm{Si}(001)$ surface.

atomic species. We assume formation of the films from the chemical elements $\mathrm{Mn}$ and $\mathrm{Si}$, i.e., the chemical potentials are determined by the cohesive energies of bulk Mn and bulk Si. For bulk Mn, we use the cohesive energy of the ground state $\alpha$-Mn, by applying a correction of $-0.07 \mathrm{eV} / \mathrm{Mn}$ (Ref. 36 ) to our calculated cohesive energy of $\gamma$-Mn (fcc structure). $E_{\mathrm{Si}(001)}^{\mathrm{surf}}$ represents the surface energy of the clean, reconstructed $\mathrm{Si}(001)$ surface, being $1.25 \mathrm{eV}$ per $(1 \times 1)$ cell in our calculations.

Results are shown in Fig. 8. As we have described earlier, ${ }^{3}$ a film with a sandwich structure of alternating Mn and $\mathrm{Si}$ layers, terminated by a Si layer, has the lowest energy of the candidate structures we investigated. In particular, it is much more stable than a film of pure Mn (upward triangles in Fig. 8), or a film of Mn capped by a Si monolayer (downward triangles of Fig. 8). These findings can be rationalized by the fact that Mn-Si bonds are stronger than the average of $\mathrm{Mn}-\mathrm{Mn}$ and $\mathrm{Si}-\mathrm{Si}$ bonds; hence the system tends to maximize the number of $\mathrm{Mn}-\mathrm{Si}$ bonds. In the sandwich films, the local coordination of a $\mathrm{Mn}$ atom is similar to the bonding in the cesium chloride (or B2) crystal structure, i.e., each Mn atoms has eight $\mathrm{Si}$ neighbors. However, due to epitaxial strain, the local environment of the Mn atoms does not have cubic symmetry, but is slightly distorted, and Mn-Si bond lengths vary by several percent within the film, being shortest in its interior and longer near the surface and interface. Negative values of $E_{\text {form }}$ in Fig. 8 indicate that the film is thermodynamically stable with respect to decomposition into a clean Si surface and bulk Mn metal. This is the case for films formed by depositing $2 \mathrm{ML}$ of $\mathrm{Mn}$ or more. Moreover, the sandwich structure is more stable than a film built up from mixed Mn-Si layers (filled square symbols in Fig. 8). Although mixed Mn-Si layers have been found to be preferable for coverages of $3 / 4 \mathrm{ML}$ or less (see Sec. II), formation of complete Mn layers becomes energetically more favorable at and above $1 \mathrm{ML}$. We take this observation as an indicator for the transition from an adsorption layer $(\theta \leqslant 3 / 4 \mathrm{ML})$ to a silicide $(\theta \geqslant 1 \mathrm{ML})$ in the B2 structure. Because of the energetic preference of the B2 film to be capped by $\mathrm{Si}$ atoms, growth of such a film requires temperatures high enough for site exchange between $\mathrm{Mn}$ and $\mathrm{Si}$ atoms to become likely.

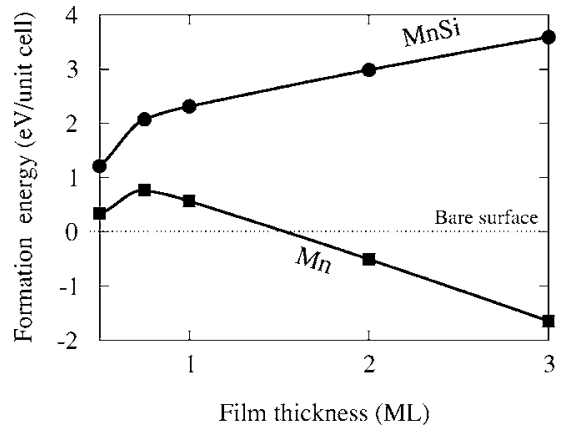

FIG. 9. Formation energy of MnSi films with B2-like structure on $\mathrm{Si}(001)$ from bulk MnSi with B20 structure (circles) or bulk Mn (squares). The surface energy of the clean $\operatorname{Si}(001) p(2 \times 2)$ reconstructed surface is considered as zero point.

Next, we consider bulk MnSi in its natural crystal structure, the B20 structure, as a source of Mn for formation of the films. This crystal structure, in which each $\mathrm{Mn}$ atom has seven bonds to $\mathrm{Si}$, is $0.25 \mathrm{eV}$ lower in energy per formula unit than the B2 structure, but cannot be grown as pseudomorphic films on $\mathrm{Si}(001)$ due to the mismatch of the two lattice structures. The formation energy versus coverage is plotted in Fig. 9 both for bulk MnSi (circles) and bulk Mn as a source for manganese (squares, same as the lowest curve in Fig. 8). The dashed line (the energy zero) is the surface energy of $\operatorname{Si}(001) p(2 \times 2)$. The formation of the pseudomorphic films from bulk MnSi with B20 structure is found to be endothermic (positive values of $E_{\text {form }}$ ), whereas formation from bulk $\mathrm{Mn}$ is exothermic for a film thickness of $2 \mathrm{ML}$ or more. Above $\theta=0.5 \mathrm{ML}$, the formation energy of the films is an almost linear function of coverage. This indicates that the interior of a 2-3 ML thick film already has properties similar to hypothetical bulk MnSi with B2 structure.

For low coverage $(\leqslant 2 \mathrm{ML})$, the films could possibly transform into three-dimensional islands and the bare $\mathrm{Si}(001)$ surface. The first step in this process is surface roughening, e.g., a film of $2 \mathrm{ML}$ thickness could decompose into regions of $1 \mathrm{ML}$ and $3 \mathrm{ML}$ thickness, driven by the energy difference

$$
\begin{aligned}
\Delta E & =\frac{1}{2}\left[E_{\mathrm{form}}(1 \mathrm{ML})+E_{\mathrm{form}}(3 \mathrm{ML})\right]-E_{\mathrm{form}}(2 \mathrm{ML}) \\
& \approx \frac{d^{2} E_{\mathrm{form}}}{d \theta^{2}}(2 \mathrm{ML}) .
\end{aligned}
$$

The formation energy of a $2 \mathrm{ML}$ film $[-0.51 \mathrm{eV} /(1 \times 1)$ cell $]$ is higher than the average formation energy for $1 \mathrm{ML}$ and 3 ML films by $0.03 \mathrm{eV} /(1 \times 1)$ cell. Since $\Delta E$ approximately equals the second derivative of the formation energy with respect to coverage, the regions of negative curvature of the curves plotted in Fig. 9 correspond to negative values of $\Delta E$. In these regions, formation of a homogenous film is unstable against decomposition into areas of different thickness. For a positive value of $\Delta E$, the formation of such a rough surface would be less favorable than a uniform film, i.e., an energy barrier against surface roughening and island formation would exist. 


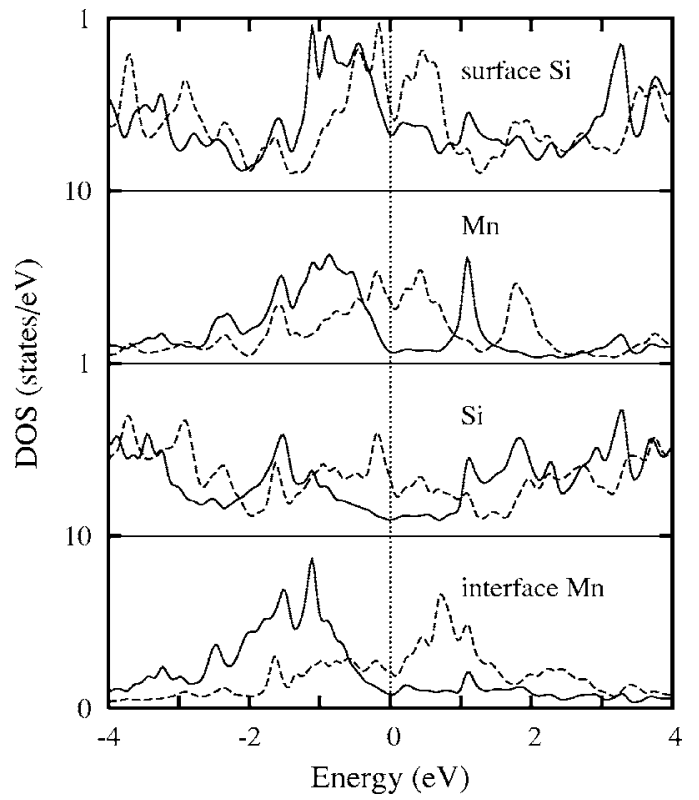

FIG. 10. Layer-resolved DOS of the FM 2(Si-Mn)/Si(001) film. The layers are shown from surface (top) to interface (bottom). Full lines show the majority spin, dashed lines the minority spin component. The considerable spin polarization at Fermi level (energy zero) is evident.

Since formation of $\mathrm{MnSi}$ multilayers $[n(\mathrm{Si}-\mathrm{Mn}) /$ $\mathrm{Si}(001)]$ with B2-like structure from bulk $\mathrm{Mn}$ is thermodynamically allowed, the magnetic properties of such structure deserve further study. Our calculations show that the 2 (Si$\mathrm{Mn}) / \mathrm{Si}(001)$ sandwich has a FM metallic ground state, being lower in energy than the interlayer AFM state, intralayer AFM one, and nonmagnetic one by 8,79 , and $188 \mathrm{meV} / \mathrm{Mn}$, respectively. Obviously, the intralayer FM coupling is rather strong, while the weak interlayer FM coupling is only about one tenth in magnitude of the former. This is due to the surface two-dimensional character of itinerant $\mathrm{Mn} 3 d-\mathrm{Si}$ $3 s 3 p$ hybridized electrons. For $3(\mathrm{Si}-\mathrm{Mn}) / \mathrm{Si}(001)$, we find that the subsurface and interface Mn layers are magnetically active, while the middle $\mathrm{Mn}$ layer becomes nearly nonmagnetic. We attribute this to a stronger covalent bond between $\mathrm{Mn}$ and $\mathrm{Si}$ in the middle layer, consistent with the shorter $\mathrm{Mn}-\mathrm{Si}$ bond length. In contrast to the weak FM interlayer coupling in the $2(\mathrm{Si}-\mathrm{Mn}) / \mathrm{Si}(001)$ sandwich mentioned above, the $3(\mathrm{Si}-\mathrm{Mn}) / \mathrm{Si}(001)$ sandwich shows an energetic preference for the magnetic moments of the subsurface $\mathrm{Mn}$ atoms and the interface $\mathrm{Mn}$ atoms to point in opposite directions. This opposite orientation of magnetic moments is lower in energy than the FM state and the nonmagnetic one by 15 and $120 \mathrm{meV}$, per magnetically active $\mathrm{Mn}$, respectively. Note that, however, the FM intralayer coupling persists also in the $3(\mathrm{Si}-\mathrm{Mn}) / \mathrm{Si}(001)$ sandwich.

In Fig. 10, we show the layer-resolved density of states of the $2(\mathrm{Si}-\mathrm{Mn}) / \mathrm{Si}(001)$ sandwich, and Fig. 11 provides the same information for the $3(\mathrm{Si}-\mathrm{Mn}) / \mathrm{Si}(001)$ sandwich. It is evident that both the Mn and Si layers have a considerable spin polarization at the Fermi level, as we reported earlier for $(\mathrm{Si}-\mathrm{Mn}) / \mathrm{Si}(001) .{ }^{3}$ In particular, the interfacial Mn layer has

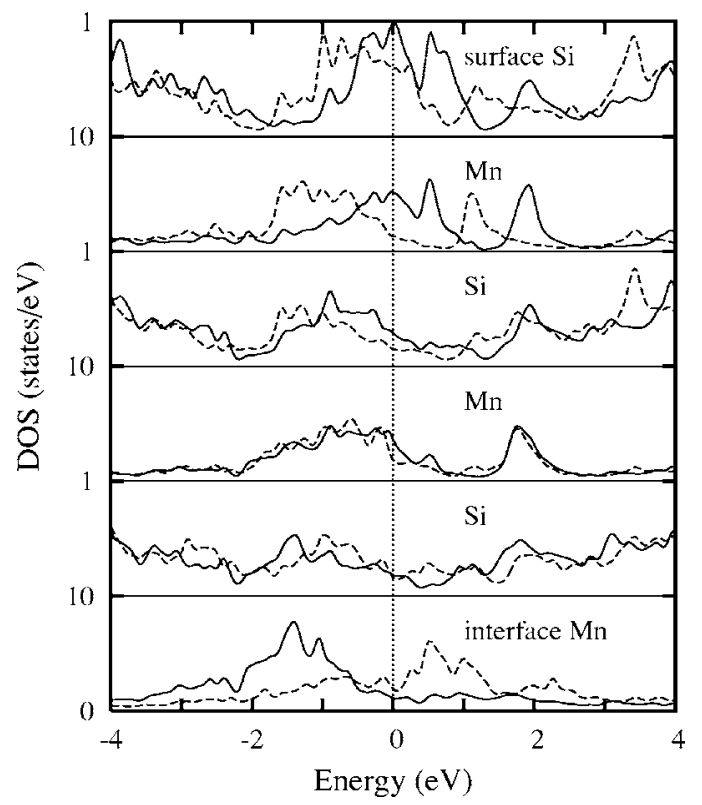

FIG. 11. Layer-resolved DOS of the $3(\mathrm{Si}-\mathrm{Mn}) / \mathrm{Si}(001)$ film with AFM interlayer spin ordering. See Fig. 10 for other notes.

a spin polarization of up to $45 \%$ in $2(\mathrm{Si}-\mathrm{Mn}) / \mathrm{Si}(001)$, and $27 \%$ in $3(\mathrm{Si}-\mathrm{Mn}) / \mathrm{Si}(001)$. These findings make the ultrathin Si-Mn sandwich films interesting candidates in the search for spintronics materials.

\section{SUMMARY AND CONCLUSIONS}

In summary, our calculations suggest the following evolution of the $\mathrm{Si}(001)$ surface under Mn deposition: The incorporation of $\mathrm{Mn}$ adatoms into subsurface interstitial sites is energetically favored, and associated with an activation barrier $(0.3 \mathrm{eV})$ low compared to on-surface diffusion barriers $(0.65 \mathrm{eV})$. Once $\mathrm{Mn}$ atoms have reached the subsurface site, their mobility is drastically reduced. Further diffusion into the bulk is energetically rather costly. For returning to the surface site the Mn atoms must overcome an energy barrier of $1.2 \mathrm{eV}$, i.e., it is a strongly activated process only taking place on a much longer time scale than incorporation. The mobility of $\mathrm{Mn}$ atoms on this time scale will take place mostly by alternating hops between subsurface interstitial sites and on-surface hollow sites, along the (110) direction of the Si dimer rows. Hence, diffusion on long time scales is expected to be anisotropic, despite the almost isotropic energy barriers for on-surface hopping.

Since $\mathrm{Mn}$ atoms at neighboring interstitial sites interact repulsively, deposition of $\mathrm{Mn}$ on a perfect $\mathrm{Si}(001)$ surface will initially lead to incorporation at randomly distributed interstitial sites, rather than to formation of two-dimensional islands. However, missing-dimer defects of the substrate surface can act as nucleation centers for MnSi clusters due to their strong binding of $\mathrm{Mn}$ adatoms. Only in the absence of these nucleation centers, two-dimensional film growth could be possible. Such preparation-dependent interface growth has been observed in experiments of Co silicide formation on $\mathrm{Si}(001) .{ }^{37}$ 
Experimentally, attempts to grow MnSi films on $\mathrm{Si}(001)$ have thus far always resulted in the formation of threedimensional islands. ${ }^{21,22}$ This is consistent with our calculations indicating that $\mathrm{MnSi} / \mathrm{Si}(001)$ does not form a wetting layer, because the formation energy of films thinner than 2 $\mathrm{ML}$ is higher than the surface energy of clean $\mathrm{Si}(001)$. Moreover, homogeneous films thinner than $2 \mathrm{ML}$ are less stable than multi-layered $\mathrm{MnSi}$ islands on clean $\mathrm{Si}(001)$. Thus, our calculations predict growth of $\mathrm{MnSi}$ on $\mathrm{Si}(001)$ in the Volmer-Weber growth mode, in agreement with experiment. Since parts of the $\mathrm{Si}(001)$ surface remain uncovered, these surface areas can act as a continuous source for Si that feeds growing MnSi islands during further deposition of $\mathrm{Mn}$.

Our calculations indicate the possibility of formation of multilayered $\mathrm{MnSi}$ islands that locally have a CsCl-like crystal structure (sandwiches of $\mathrm{Mn}$ and $\mathrm{Si}$ layers) as possible epitaxial structures. In these sandwiches, the Mn atoms near the surface and interface have sizable magnetic moments that couple ferromagnetically within the layers. The layerresolved electronic density-of-states indicates a high degree of spin polarization at the Fermi level, up to $45 \%$ and $27 \%$ for two or three Si-Mn layers, respectively. Thus far, there are no measurements or reliable estimates of the Curie temperatures of these structures. If their Curie temperatures are found to be sufficiently high, MnSi islands grown on $\mathrm{Si}(001)$ might become a useful component of spintronics devices.

\section{ACKNOWLEDGMENTS}

This work was supported by the Deutsche Forschungsgemeinschaft through SFB 290.
${ }^{1}$ G. Prinz and J. Krebs, Appl. Phys. Lett. 39, 397 (1981).

${ }^{2}$ G. Prinz, Science 282, 1660 (1998).

${ }^{3}$ H. Wu, M. Hortamani, P. Kratzer, and M. Scheffler, Phys. Rev. Lett. 92, 237202 (2004).

${ }^{4}$ H. Wu, P. Kratzer, and M. Scheffler, Phys. Rev. B 72, 144425 (2005).

${ }^{5}$ W. Zhu, H. H. Weitering, E. G. Wang, E. Kaxiras, and Z. Zhang, Phys. Rev. Lett. 93, 126102 (2004).

${ }^{6}$ G. M. Dalpian, A. J. R. da Silva, and A. Fazzio, Phys. Rev. B 68, 113310 (2003).

${ }^{7}$ G. M. Dalpian, A. J. R. da Silva, and A. Fazzio, Surf. Sci. 688, 566 (2004)

${ }^{8}$ A. J. R. da Silva, A. Fazzio, and A. Antonelli, Phys. Rev. B 70, 193205 (2004).

${ }^{9}$ M. Bolduc, C. Awo-Affouda, A. Stollenwerk, M. B. Huang, F. G. Ramos, G. Agnello, and V. P. LaBella, Phys. Rev. B 71, 033302 (2005).

${ }^{10}$ A. P. Horsfield, S. D. Kenny, and H. Fujitani, Phys. Rev. B 64, 245332 (2001).

${ }^{11}$ S. Higai and T. Ohno, Phys. Rev. B 62, R7711 (2000).

${ }^{12}$ M. M. R. Evans, J. C. Glueckstein, and J. Nogami, Phys. Rev. B 53, 4000 (1996).

${ }^{13}$ T. Nagao, S. Ohuchi, Y. Matsuoka, and S. Hasegawa, Surf. Sci. 419, 134 (1999).

${ }^{14}$ G. Ctistis, U. Deffke, J. Paggel, and P. Fumagalli, J. Magn. Magn. Mater. 240, 420 (2002).

${ }^{15}$ G. Ctistis, U. Deffke, K. Schwinge, J. J. Paggel, and P. Fumagalli, Phys. Rev. B 71, 035431 (2005).

${ }^{16}$ S. M. Shivaprasad, C. Anandan, S. Azatyan, Y. Gavriljuk, and V. Lifshits, Surf. Sci. 382, 258 (1997).

${ }^{17}$ S. G. Azatyan, M. Iwami, and V. G. Lifshits, Surf. Sci. 589, 106 (2005)

${ }^{18}$ S. Kawamoto, M. Kusaka, M. Hirai, and M. Iwami, Surf. Sci. 242, 331 (1991).

${ }^{19}$ A. Kumar, M. Tallarida, M. Hansmann, U. Starke, and K. Horn, J. Phys. D 37, 1083 (2004).
${ }^{20}$ J. Wang, M. Hirai, M. Kusaka, and M. Iwami, Appl. Surf. Sci. 113-114, 53 (1997).

${ }^{21}$ H. Lippitz, J. J. Paggel, and P. Fumagalli, Surf. Sci. 575, 307 (2005).

${ }^{22}$ M. R. Krause, A. Stollenwerk, M. Licurse, and V. P. LaBella, J. Vac. Sci. Technol. A 24, 1480 (2006).

${ }^{23}$ Y. Zhang, Q. Jiang, D. J. Smith, and J. Drucker, J. Appl. Phys. 98, 033512 (2005).

${ }^{24}$ P. Blaha, K. Schwarz, G. K. H. Madsen, D. Kvasnicka, and J. Luitz, WIEN2k, an Augmented Plane Wave+Local Orbitals Program for Calculating Crystal Properties (Technical University, Vienna, Austria, 2001).

${ }^{25}$ J. P. Perdew, K. Burke, and M. Ernzerhof, Phys. Rev. Lett. 77, 3865 (1996).

${ }^{26}$ M. Eder, J. Hafner, and E. G. Moroni, Phys. Rev. B 61, 11492 (2000).

${ }^{27}$ E. Sjöstedt, L. Nordström, and D. J. Singh, Solid State Commun. 114, 15 (2000).

${ }^{28}$ A. Ramstad, G. Brocks, and P. J. Kelly, Phys. Rev. B 51, 14504 (1995).

${ }^{29}$ F. Beeler, O. K. Andersen, and M. Scheffler, Phys. Rev. B 41, 1603 (1990).

${ }^{30}$ H. J. W. Zandvliet, Surf. Sci. 377-379, 1 (1997).

${ }^{31}$ S. C. Erwin and A. G. Petukhov, Phys. Rev. Lett. 89, 227201 (2002).

${ }^{32}$ B. D. Yu, Y. Miyamoto, O. Sugino, T. Sasaki, and T. Ohno, Phys. Rev. B 58, 3549 (1998).

${ }^{33}$ S. Higai and T. Ohno, Appl. Surf. Sci. 166, 149 (2000).

${ }^{34}$ E. R. Weber, Appl. Phys. A: Solids Surf. 30, 1 (1983).

${ }^{35}$ D. Gilles, W. Bergholz, and W. Schroeter, J. Appl. Phys. 59, 3590 (1986).

${ }^{36}$ D. Hobbs and J. Hafner, J. Phys.: Condens. Matter 13, L681 (2001).

${ }^{37}$ H. L. Meyerheim, U. Döbler, and A. Puschmann, Phys. Rev. B 44, 5738 (1991). 